\title{
DESENVOLVIMENTO DE UMA PARAMETRIZAÇÃO DO EFEITO DE RAJADAS ASSOCIADAS A SISTEMAS CONVECTIVOS NOS FLUXOS DE CALOR NA SUPERFÍCIE UTILIZANDO MODELAGEM DE ENSEMBLE DE NUVENS
}

\author{
AURÉLIO WILDSON TEIXEIRA DE NORONHA, ALEXANDRE ARAÚJO COSTA E ANTÔNIO \\ CARLOS SANTANA DOS SANTOS
}

\author{
Universidade Estadual do Ceará (UECE), Fortaleza, CE, Brasil \\ awildson@gmail.com, alexandrearaujoc@gmail.com,dr.acss@gmail.com
}

Recebido Junho de 2012 - Aceito Abril de 2013

\begin{abstract}
RESUMO
Sistemas convectivos precipitantes produzem rajadas a partir de suas correntes descendentes, quando estas atingem a superfície. Tais rajadas têm a capacidade de amplificar os fluxos superficiais de calor sensível e latente, com possíveis consequências para a própria evolução futura da convecção. Modelos de Circulação Geral da Atmosfera e até modelos regionais, ainda que tenham se tornado mais eficientes e robustos, ainda estão muito longe de representarem adequadamente (explicitamente) as nuvens e, portanto, o efeito de intensificação dos fluxos por rajadas não é por eles simulado. Na verdade, tal efeito sequer é parametrizado, na maioria dos casos. No presente trabalho, um CEM (Cloud Ensemble Model), alimentado por forçante de grande escala característico de condições de convecção tropical, foi usado para encontrar uma parametrização que represente a intensificação dos fluxos de calor da superfície por rajadas, por meio de um fator, que é função da taxa de precipitação, do vento resolvido e da resolução espacial do modelo de grande escala. $\mathrm{O}$ fator de intensificação possui correlações significativas com o vento resolvido $(-0,67 \leq \rho \leq-0,46)$ e a taxa de precipitação $(0,65 \geq \rho \geq 0,4)$, que são usadas como variáveis de entrada para calculá-lo. A parametrização foi encontrada a partir do ajuste de parâmetros utilizando os dados de saída das simulações com o CEM. Palavras-chaves: Modelo de Ensemble de Nuvens; Parametrização da Intensificação de fluxos; Experimento TOGA-COARE.
\end{abstract}

\begin{abstract}
DEVELOPMENT OF A PARAMETERIZATION OF THE EFFECTS OF GUSTS ASSOCIATED WITH CONVECTIVE SYSTEMS IN THE SURFACE HEAT FLUXES USING A CLOUD ENSEMBLE MODEL

Precipitating convective systems produce gusts from downdrafts as they reach the surface. Those gusts are able to intensify the surface fluxes of sensible and latent heat, with possible consequences to the further evolution of deep convection itself. Despite advances in resolution and in the representation of physical processes, General Circulation Models and even regional models are still very far from representing clouds adequately (i.e., explicitly) and therefore surface flux enhancement by gusts is not simulated by them. In fact, such effect is not usually even parameterized. In this work, a CEM (Cloud Ensemble Model), driven by a prescribed large-scale forcing corresponding to tropical convective conditions, was used to find a parameterization that evaluates surface heat flux enhancement, as a function of precipitation rate, resolved wind and of the resolution of the larger-scale model. The enhancement factor is well correlated with both the resolved wind $(-0.67 \leq \rho \leq-0.46)$ and the precipitation rate $(0.65 \geq \rho \geq 0.4)$, which were used as input variables for calculating it. The parameterization equation was obtained by fitting the data from the CEM simulations.
\end{abstract}

Keywords: Cloud Ensemble Model, Flux Enhancement Parameterization, TOGA-COARE 


\section{INTRODUÇÃO}

Modelos de circulação geral da atmosfera (MCGAs), especialmente em simulações climáticas, tem seu espaçamento de grade (resolução espacial) limitada tipicamente a algo da ordem de centena de quilômetros. Portanto, os processos na escala de subgrade não são resolvidos, e mesmo seu efeito coletivo na grande escala pode não estar sendo bem representado. No caso dos MCGAs isto inclui processos de mesoescala, como a interação do fluido atmosférico com a topografia e a turbulência na microescala.

Neste contexto, o quarto relatório do IPCC cita que os modelos de circulação geral da atmosfera ainda possuem elevados níveis de incerteza na capacidade de representar os processos físicos envolvendo nuvens e seus respectivos efeitos no planeta (Tremback et al., 2007). As nuvens são um componente crucial do sistema climático, pois produzem efeitos consideráveis no balanço radiativo, tanto de onda curta, quanto de onda longa, assim são peças fundamentais no transporte de energia na atmosfera; ao liberarem calor latente em função dos processos de mudança de fase da água. Sobre a superfície do planeta, especialmente os oceanos, os fluxos de calor latente e sensível têm um papel importante para os processos hidrológicos, atmosféricos e para o ciclo de energia; contribuindo com cerca de $30 \%$ anuais do balanço energético global causando profundos impactos no clima global e regional (Peixoto e Oort, 1991; Wu e Guimond, 2006; Wu e Li, 2008).

Um efeito pouco investigado das nuvens convectivas é a sua influência sobre os fluxos superficiais de calor. Especialmente se o vento de grande escala for pouco intenso, os fluxos de calor latente e sensível sofrem grandes modificações na presença de nuvens convectivas precipitantes. O mecanismo de intensificação dos fluxos de calor (Costa et al., 2001; Redelsperger et al., 2000) é relativamente simples e é descrito a seguir. A precipitação, ao evaporar, tende a resfriar a massa de ar sob a base da nuvem. Esta tende a se acelerar para baixo, produzindo correntes descendentes ("downdrafts"), cuja intensidade pode ser bastante significativa. Tais correntes, ao se aproximarem da superfície, são defletidas para a horizontal, produzindo rajadas. Especialmente se o vento de grande escala for pequeno, as rajadas podem ser responsáveis por valores elevados dos fluxos superficiais de calor sensível e latente.

Como apontado por Costa et al. (2001), esses fluxos podem ser significativamente intensificados pelas rajadas, cuja intensidade depende da precipitação. Redelspeger et al. (2000) apresentou, com base em observações do Tropical Ocean Global Atmosphere - Coupled Ocean - Atmosphere Experiment (TOGA-COARE), uma parametrização simples em que uma a intensificação dos fluxos é função apenas da taxa de precipitação.
No entanto, a resolução espacial de um modelo de grande escala em que tal efeito for parametrizado também é um fator importante e deveria entrar no seu equacionamento. Afinal, para modelo de circulação geral e modelo de mesoescala com maior resolução, tais rajadas começam a ser resolvidas, isto é, na representação desses fluxos, o fator de intensificação deve ser, tanto maior, quanto maior for o espaçamento de grade do modelo. Zeng et al. (2002) mostra uma dependência da intensificação dos fluxos, quando leva-se em conta a resolução espacial durante os experimentos COARE I e COARE II. Esses experimentos são uma referência a dois períodos do conjunto experimental TOGA-COARE: COARE I compreende o período de 10 a 17 de dezembro de 1992 e COARE II o período de 19 a 27 de dezembro de 1992.

Wu e Guimond (2006) mostram que a intensificação de fluxos de calor na superfície ainda não é incorporada na grande maioria dos modelos de circulação geral, entretanto, os mesmos comentam que as circulações tropicais são sensíveis aos efeitos de rajadas nos fluxos de superfície sob baixos valores da velocidade do vento. Assim, surge a necessidade no desenvolvimento de parametrizações adequadas deste efeito e sua implementação em modelos.

Nesse contexto, o modelo de "ensemble" (ou conjunto) de nuvens, Cloud-Ensemble-Model, (CEM) é uma ferramenta poderosa para o estudo da convecção profunda e sua interação com o ambiente de grande escala, podendo ser utilizado para a construção de parametrizações físicas dos efeitos das nuvens. Um CEM resolve nuvens convectivas, sem a necessidade de uma parametrização de convecção, calculando os processos dinâmicos das nuvens, como por exemplo, o entranhamento e desentranhamento dos fluxos de massa, a formação da precipitação, a interação das nuvens com a radiação, etc. O CEM é um modelo numérico de tempo capaz de resolver os processos dinâmicos da atmosfera em escala de nuvens, com resolução espacial com cerca de $1 \mathrm{~km}$, num domínio espacial relativamente grande o suficiente, para abrigar várias nuvens e seus respectivos ciclos de vida (Moncrieff et al., 1997; Randall et al., 1996; Wu e Li, 2008). Os CEMs têm sido utilizados para entender processos dinâmicos atuantes em escala de nuvens, dentre os quais se destacam a formação da precipitação, sua influência sobre a radiação entre outras variáveis (Grabowski et al., 1996; Randall et al., 1996; Grabowski et al., 1998; Grabowski et al., 1999; Redelsperger et al., 2000; Costa, 2004a, 2004b; Noronha et al., 2011; Pinheiro e Costa, 2013).

Uma parametrização do efeito da intensificação dos fluxos de calor inserida em um MCGA pode contribuir num aumento da precipitação acumulada em escala global, principalmente sobre a região oceânica tropical. A precipitação é um produto das nuvens, a formação de nuvens depende de condições atmosféricas e do saldo de vapor d'água e térmico 
da atmosfera. $\mathrm{O}$ vapor d'água atmosférico tem como principal fonte os fluxos de calor latente. Portanto, aumentar os fluxos de calor removidos da superfície pode contribuir expressivamente no aumento das estimativas de precipitação em MCGAs.

O objetivo geral é mostrar que a intensificação dos fluxos de superfície por rajadas, por meio de um fator, está correlacionado com a taxa de precipitação (mostrando também dependência em relação à velocidade do vento de grande escala próximo à superfície e o espaçamento de grade de um modelo de grande escala) e utiliza-se dessa relação para apresentar uma parametrização desse fator, que represente o efeito das rajadas produzidas por nuvens convectivas precipitantes em MCGA e modelos atmosféricos regionais.

\section{MATERIAL E MÉTODOS}

\subsection{Modelo numérico e dados}

Neste trabalho foi utilizada uma versão modificada do modelo RAMS 6.0 (Regional Atmospheric Modelling System), que foi adaptada para servir como um CEM. A adaptação consistiu na adição de um nudging não local para o momentum ao modelo (Costa et al., 2001; Grabowski et al., 1996). O nudging não local tem a expressão:

$$
\begin{aligned}
& \left.\frac{\partial u}{\partial t}\right|_{F G E}=-\frac{\bar{u}-u_{0}}{\tau}, \\
& \left.\frac{\partial v}{\partial t}\right|_{F G E}=-\frac{\bar{v}-v_{0}}{\tau},
\end{aligned}
$$

onde $\bar{u}$ e $\bar{v}$ são os componentes médios zonal e meridional do vento, $u_{0}$ e $v_{0}$ são os valores de vento das sondagens e $\tau$ é o tempo de relaxamento. Este tipo de nudging mantém a média da velocidade de vento do CEM muito próximo das sondagens e, permite a propagação de padrões de menor escala no domínio do modelo.

O modelo contou com a adição de forçantes de grande escala de vento zonal e meridional; advecção de temperatura e umidade a partir de dados do experimento TOGA (Tropical Ocean Global Atmosphere) - COARE (Coupled Ocean Atmosphere Research Experiment), coletados durante o IOP (Intensive Observing Period) no período de 01/11/1992 0000 UTC a 28/02/1993 0000 UTC. A partir destes dados foi realizado o cálculo das médias durante todo o período do IOP e aplicado, no CEM, o chamado nudging não-local, descrito por Grabowski et al. (1996), Costa et al. (2001), Pinheiro e Costa (2013), para as variáveis da velocidade do vento zonal e meridional no conjunto das simulações.

As parametrizações escolhidas para os experimentos numéricos foram a de radiação de onda curta e onda longa de Chen e Cotton (1987); turbulência do tipo anisotrópica de
Smagorinsky (1963) e microfísica totalizada de um momento com sete classes de hidrometeoros, sendo os mesmos água de nuvem, água de chuva, neve, pristine, granizo, graupel e agregados de gelo de Walko et al. (1995).

\subsection{Formulação da parametrização}

Como discutido, o fator de intensificação dos fluxos de calor está relacionado com a intensidade da rajada gerada pelas nuvens convectivas. Segundo Costa (2000) e Costa et al. (2001), quando nuvens convectivas estão presentes na grade de modelos numéricos sob valores pequenos de velocidade do vento de grande escala, há uma diferença significativa entre o módulo da velocidade média de grande escala $|\bar{V}|$ e a média do módulo do vetor velocidade em escala de subgrade $\overline{|V|}$. Assim, sob essas condições é verificada uma intensificação nos fluxos superficiais.

Subdividindo-se o domínio maior de um modelo de grande escala (como um MCGA) em diversos subdomínios (nas direções $x$ e $y$, por exemplo), o módulo da velocidade média, ou seja, o valor resolvido do vento nessa caixa, está relacionado com os valores em escala de subgrade conforme a Equação 3:

$$
|\bar{V}|=\sqrt{\left(\frac{1}{N_{x} N_{y}} \sum_{i=1}^{N_{x}} \sum_{j=1}^{N_{y}} u_{i, j}\right)^{2}+\left(\frac{1}{N_{x} N_{y}} \sum_{i=1}^{N_{x}} \sum_{j=1}^{N_{y}} v_{i, j}\right)^{2}},
$$

em que $\boldsymbol{V}$ se refere ao vetor velocidade, e às suas componentes zonal e meridional e $N_{x}$ e $N_{y}$ são os números de subdomínios nas duas direções.

Por sua vez, a média do módulo da velocidade do vento, que deveria ser a variável utilizada no cálculo dos fluxos, é dada, ao se considerar a variabilidade em escala de subgrade, por:

$$
\overline{|V|}=\frac{1}{N_{x} N_{y}} \sum_{i=1}^{N_{x}} \sum_{j=1}^{N_{y}} \sqrt{u_{i, j}^{2}+v_{i, j}^{2}}
$$

Define-se que o fator de intensificação será a razão entre as velocidades das Equações 3 e 4, subtraída de um e assume-se que o mesmo é função do vento de grande escala e da taxa de precipitação, tal que:

$$
\varepsilon(|\bar{V}|, P)=\frac{\overline{|V|}}{|\bar{V}|}-1
$$

Visto de outro modo, pode-se considerar que, num modelo de maior escala, a intensidade do vento que deve ser utilizada nos cálculos dos fluxos de superfície, particularmente em fórmulas aerodinâmicas, tem de ser modificado para:

$$
V^{*}=V[1+\varepsilon]
$$

em que $V^{*}$ é o vento modificado, incluindo a ação das rajadas.

A taxa de precipitação foi calculada usando um esquema de diferenças finitas centrada no tempo, ao invés 
de usar valores instantâneos, como objetivo de suavizar a dependência temporal dessa variável, facilitando a obtenção de uma equação do parâmetro de intensificação dos fluxos em sua função.

\subsection{Experimentos numéricos}

Foram realizadas cinco simulações que diferem entre si por uma translação do perfil vertical do vento zonal médio observado durante o TOGA-COARE IOP. Os perfis de vento zonal foram movidos de modo que os novos mantivessem a forma do perfil da sondagem, mudando-se apenas os valores. Desta forma, seis perfis de velocidade foram obtidos, sendo que a intensidade do vento à superfície variou entre $0 \mathrm{~m} / \mathrm{s}$ a $5 \mathrm{~m} / \mathrm{s}$ no nível da superfície, como é ilustrado na Figura 1.

Cada simulação teve a duração de setecentos e vinte horas (01 de Novembro de 1992 as 0000 UTC a 01 de Dezembro de 1992 as 0000 UTC), sendo mantida uma temperatura da superfície do mar constante de 302,54 K.

O domínio de simulação tem uma estrutura bidimensional contendo 1024 pontos na direção horizontal e 70 níveis na vertical. O espaçamento de grade horizontal é de $1 \mathrm{~km}$ e, na vertical, a resolução é variável, com espaçamento de $80 \mathrm{~m}$ próximo à superfície até $600 \mathrm{~m}$ próximo ao topo do domínio, posicionado em $21361 \mathrm{~m}$. O modelo é integrado a cada 10 segundos, com saída horária, e a integração no tempo da radiação é de 600 segundos.

No pós-processamento das simulações, foi realizada uma filtragem dos dados de maneira que os valores nulos da taxa de precipitação (centrada no tempo), condição que não interessa ao presente estudo, e os valores acima de 6 para o fator de intensificação dos fluxos ("outliers") foram omitidos no conjunto dos dados.

\section{RESULTADOS E DISCUSSÕES}

\subsection{Quase-equilíbrio}

A fim de que o CEM possa ser usado no desenvolvimento de parametrizações, alimentado por uma forçante advectiva constante, é necessário verificar se ele atingiu um estado de "quase-equilíbrio", isto é, se as variáveis dinâmicas e termodinâmicas oscilam em torno de um valor médio, não havendo tendência continuada de redução ou aumento. Isto acontece, quando a forçante advectiva, que produz resfriamento e umedecimento de grande escala, é contrabalançada pela ação da convecção, que, graças às nuvens precipitantes, induz aquecimento (por liberação de calor latente) e secagem (por remoção da água do ambiente pela precipitação).

Em concordância com a teoria de Arakawa e Schubert (1974), o CEM foi capaz de reproduzir os efeitos esperados de desenvolvimento da convecção e consumo de vapor d'água fornecidos pela grande escala ao modelo. Quando há produção de precipitação, também há uma redução de Convective Available Potential Energy (CAPE) nas simulações (Figura 3).

Neste trabalho, o CEM, em todas as simulações, apresentou comportamento que indica a tendência a estados de quase-equilíbrio, ou seja, indicando que o modelo tem a capacidade de responder como esperado às forçantes de grande escala impostas durante a simulação.

Deve ser ressaltado, no entanto, que cada simulação apresentou diferenças quanto ao tempo necessário para

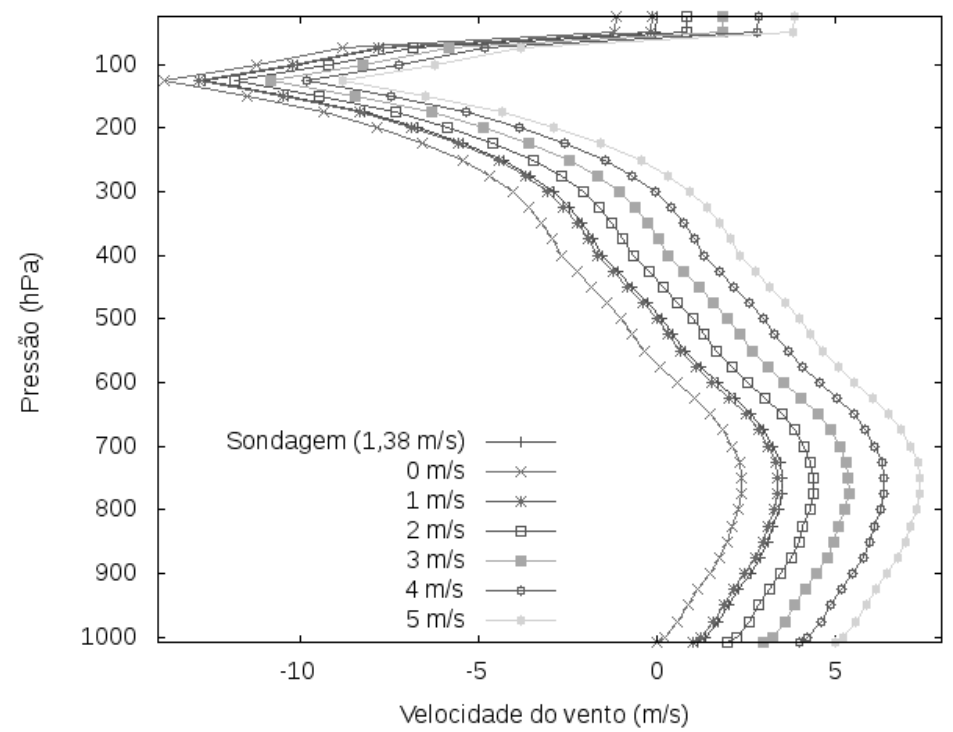

Figura 1 - Perfis verticais da velocidade do vento zonal para o nudging não local assumidos no conjunto de simulações. A legenda do gráfico identifica o valor de velocidade de vento no primeiro nível de cada perfil. 
atingir esse estado como é apresentado na Figura 2, em que é apresentada a evolução temporal da média sobre o domínio da energia potencial convectiva disponível.

A quantidade energética no início da simulação apresenta uma tendência de queda, mas depois de um tempo a variável tende a um comportamento de quase-equilíbrio. Os tempos diferentes do CEM para atingir estados de quase-equilíbrio, devem-se aos forçantes de grande escala que são constantes no tempo, diferente do que foi feito em outros trabalhos com configurações semelhantes e forçantes que variam no tempo das sondagens do TOGA-COARE (Costa et al., 2001; Pinheiro e Costa, 2013).

Os fluxos de calor latente e sensível (Figura 4) partem de valores menores e tipicamente após poucos dias de simulação tendem a oscilar em torno de um valor praticamente constante. As simulações com menores valores do vento de grande escala próximo à superfície foram aquelas que levaram mais tempo a chegar ao quase-equilíbrio.

A Tabela 1 mostra os valores médios dos fluxos de calor e da taxa de precipitação após o período considerado transiente (primeiras 360 horas de simulação). O vento de grande escala imposto como forçantes ao modelo exerce um papel importante, na medida em que modula os fluxos de superfície e, em função principalmente de uma maior evaporação no caso de ventos superficiais maiores, também modifica o balanço de água e a taxa de precipitação. Os fluxos de calor latente aumentam seu valor significativamente para a simulação V5, em comparação à simulação V0 (aumento de cerca de 80\%). Consequentemente, também há um aumento significativo na taxa de precipitação nas simulações com maiores valores do vento de superfície, quando comparadas à simulação V0, chegando a mais de $20 \%$ no caso V5.

\subsection{Exemplo de intensificação dos fluxos por um sistema convectivo}

Como mostrado na Tabela 1, os fluxos de calor latente e sensível aumentam significativamente com o aumento do valor da velocidade do vento de grande escala imposto ao CEM, mas essas grandezas estão longe de serem diretamente
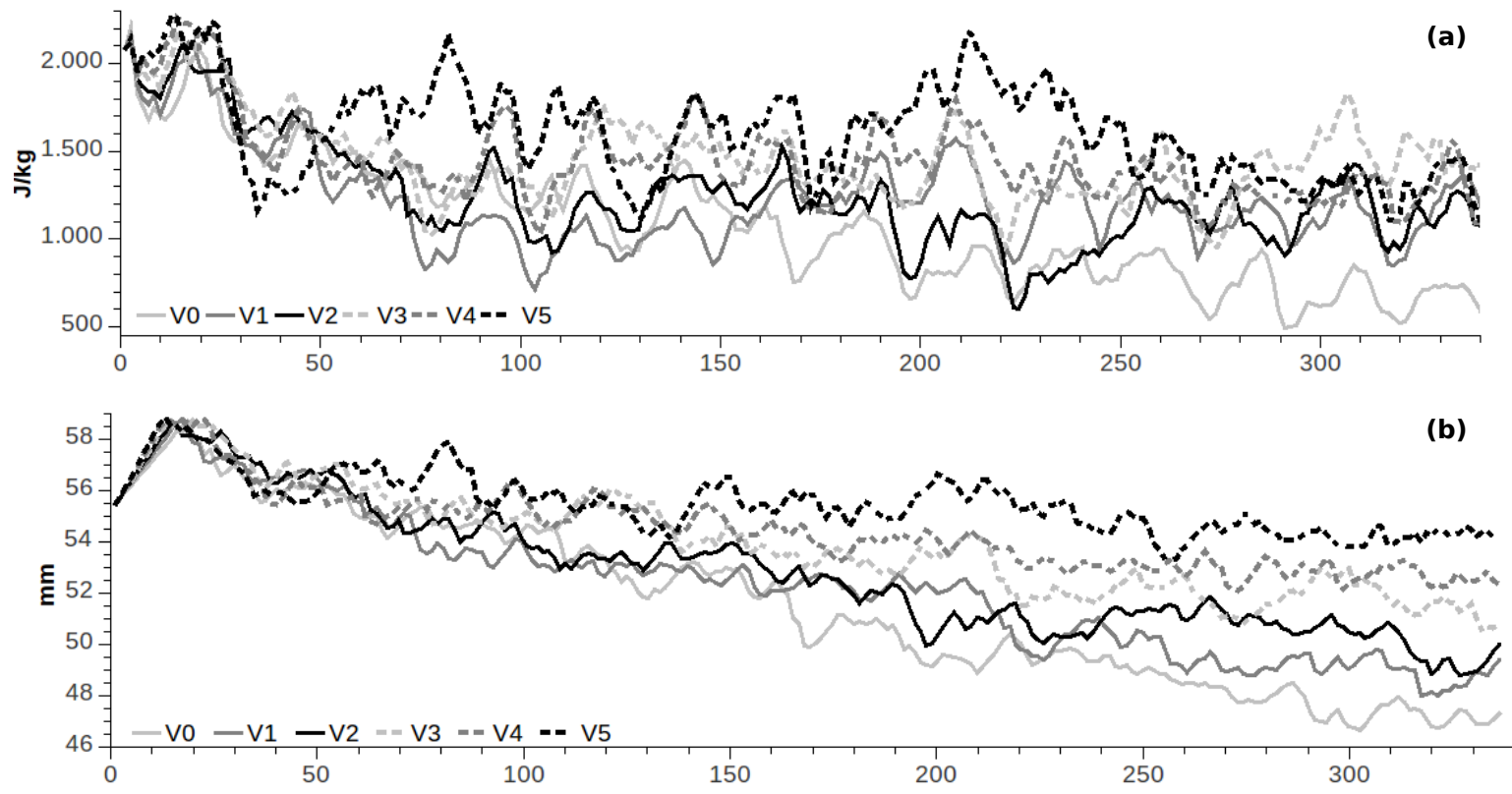

(b)

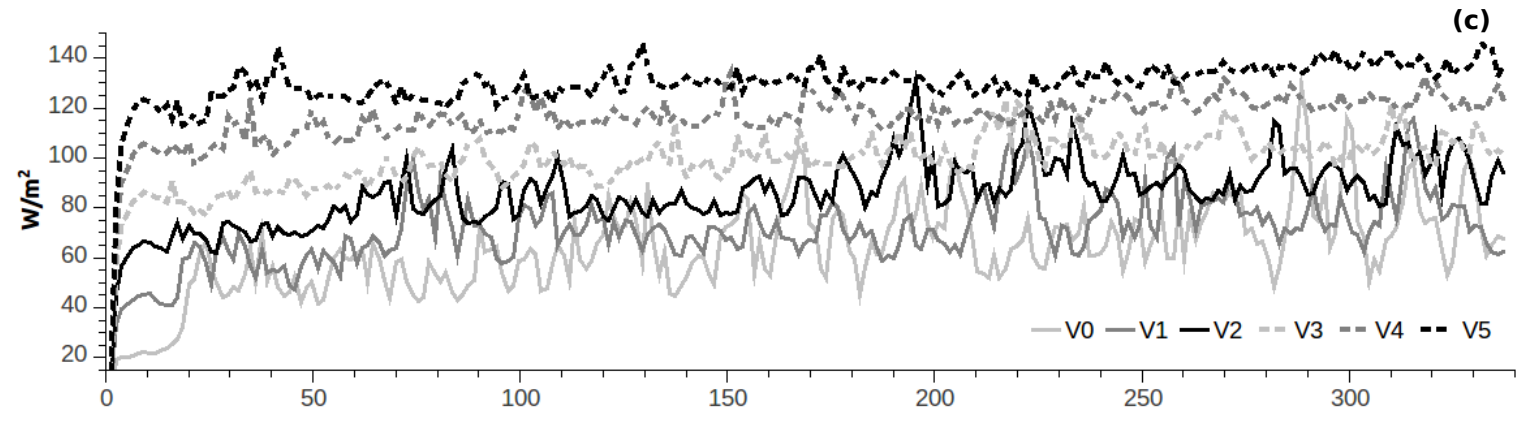

Figura 2 - Energia Potencial Convectiva Disponível (a), Água Precipitável (b) e Fluxo de Calor Latente (c) no período transiente dos experimentos numéricos do CEM. As horas simuladas dos experimentos são mostradas no eixo x entre 1 (01/11/1992 00:00 UTC) a 340 (15/11/1992 00:00 UTC). 

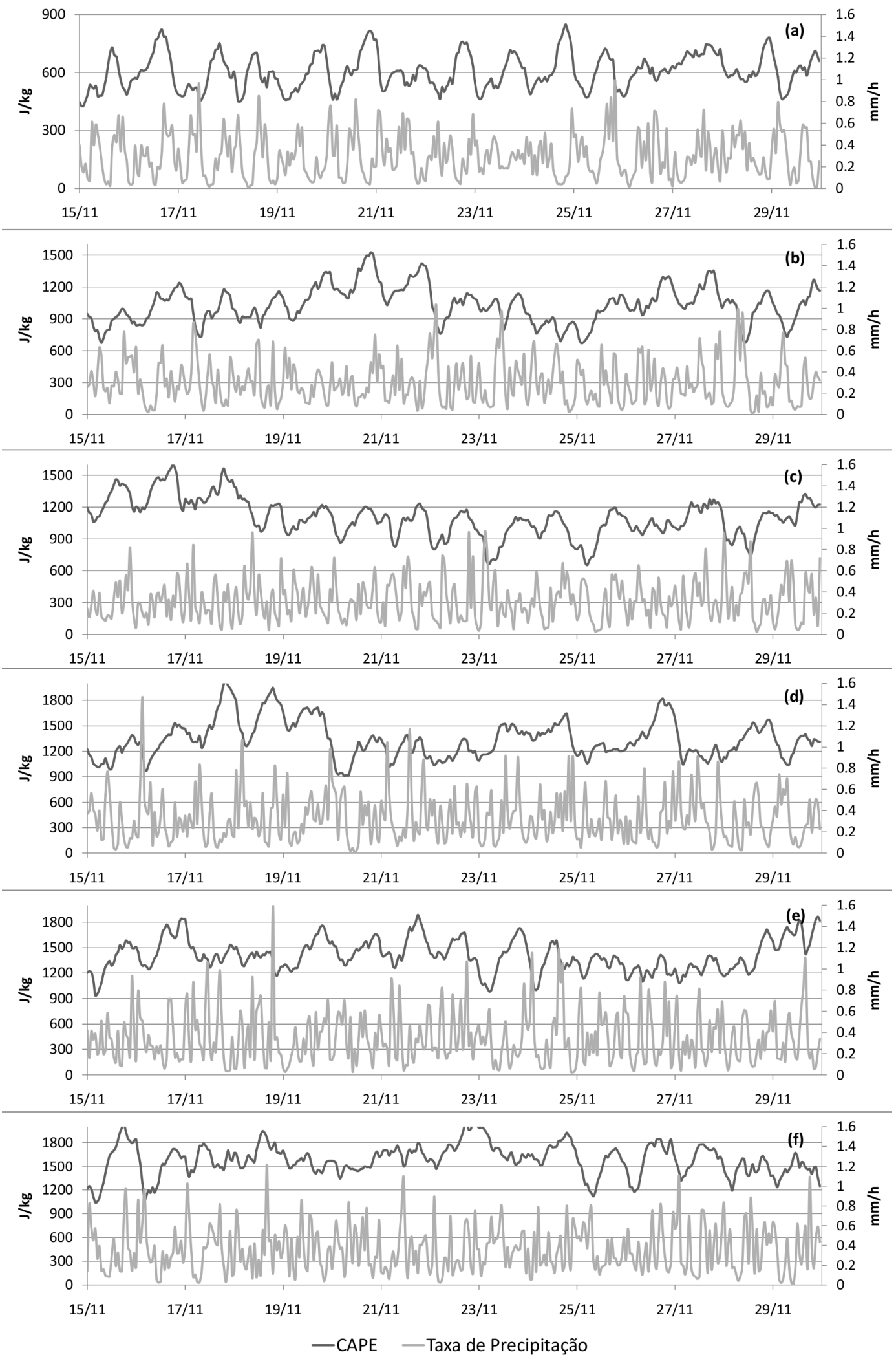

Figura 3 - Taxa de Precipitação $(\mathrm{mm} / \mathrm{h})$ e Energia Potencial Convectiva Disponível ( $/ \mathrm{kg}$ ) sem o período transiente nos experimentos do CEM: V0 (a), V1 (b), V2 (c), V3 (d), V4(e), V5 (f). Os dias simulados dos experimentos são mostrados no eixo x entre 15 a 30 de Novembro de 1992. 


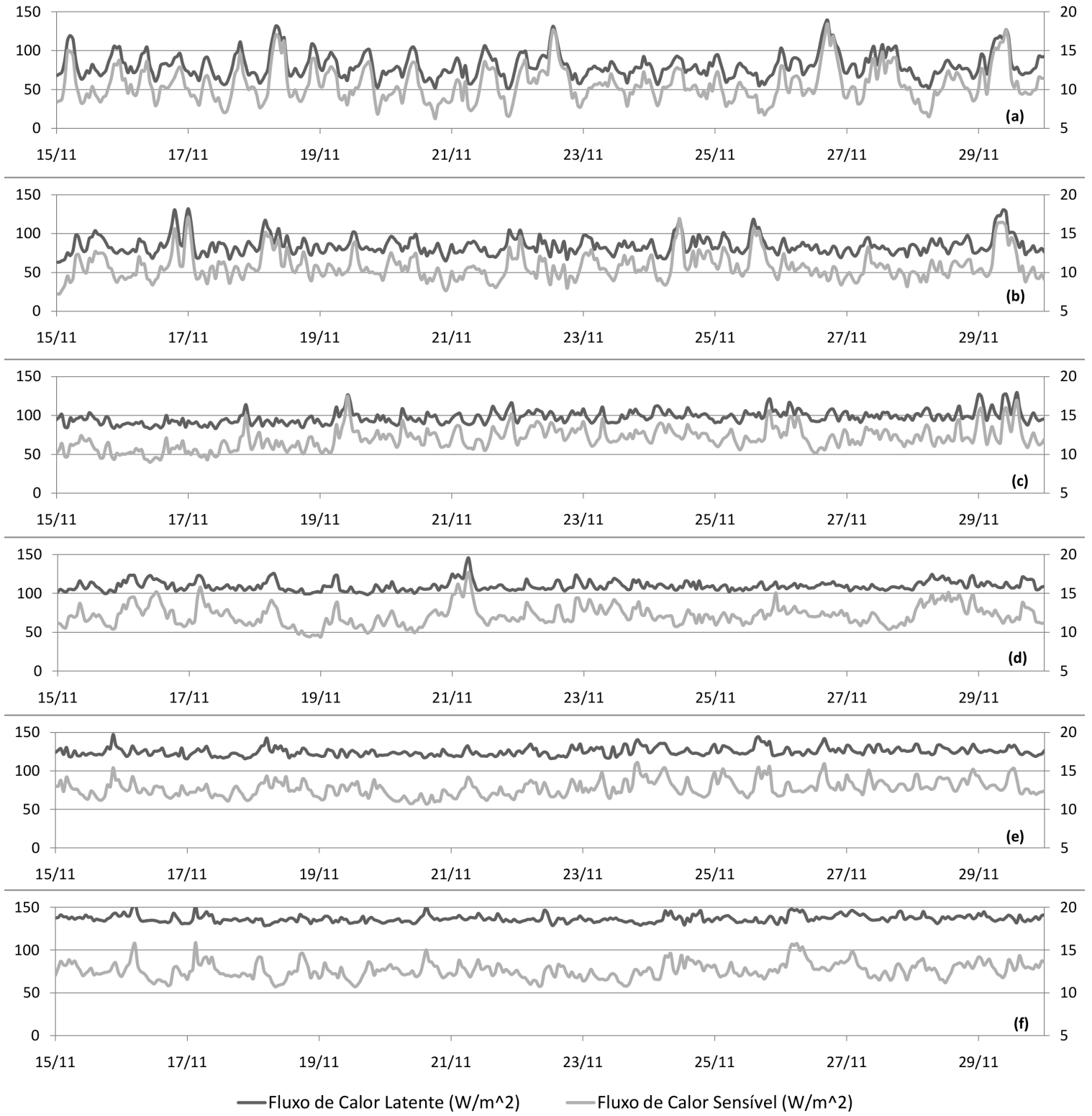

Figura 4 - Fluxos de Calor Sensível e Latente (W/m2) para cada simulação sem o período transiente: V0 (a), V1 (b), V2 (c), V3 (d), V4(e), V5 (f). Os dias simulados dos experimentos são mostrados no eixo x entre 15 a 30 de Novembro de 1992.

proporcionais, como geralmente assumido em modelos de grande escala. Principalmente, quando o vento de grande escala é fraco, os fluxos de calor são dominados pelas rajadas dos sistemas convectivos. Para evitar que esses fluxos caiam a zero ou a valores irrealisticamente pequenos, os modelos de maior escala impõem um vento mínimo, isto devido, a uma restrição ao coeficiente de arrastro; dado que o coeficiente de arrastro é uma função inversamente proporcional à velocidade do vento (Bradley et al., 1991; Mitsuta e Tsukamoto, 1978).

Para exemplificar o papel significativo das rajadas, na Figura 5 é apresentado um corte vertical da simulação do grupo
V0 na hora 636, numa parte do domínio, de 100 km de extensão. No painel 5a, é mostrada a razão de mistura de condensado total (incluindo todas as espécies de água nas fases líquida e sólida), indicando a formação de um sistema convectivo, bem como, os vetores de vento. Padrões básicos são evidenciados como a existência da bigorna, as frentes de rajadas de fronte ao sistema, intrusão de ar quente por convecção forçada para dentro, entranhamento ao longo da vertical (baixo para cima) e desentranhamento de ar no topo do sistema. O painel $5 \mathrm{~b}$ mostra a taxa de precipitação e o painel $5 \mathrm{c}$, o vento zonal, com valores maiores em módulo, mas de sinal invertido, em torno do máximo 
Tabela 1 - Cálculo das médias dos fluxos de calor latente e sensível e seus respectivos percentuais de aumento em relação ao grupo de simulação V0.

\begin{tabular}{cccc}
\hline & \multicolumn{3}{c}{ Valor médio em todo domínio espacial e temporal } \\
Simulação & $\begin{array}{c}\text { Fluxo de Calor Latente } \\
\left(\mathbf{W} / \mathbf{m}^{2}\right)-\text { Crescimento } \\
\text { em relação a V0 (\%) }\end{array}$ & $\begin{array}{c}\text { Fluxo de Calor Sensível } \\
\left(\mathbf{W} / \mathbf{m}^{2}\right) \text { - Crescimento } \\
\text { em relação a V0 (\%) }\end{array}$ & $\begin{array}{c}\text { Taxa de } \\
\text { Precipitação } \\
(\mathbf{m m} / \mathbf{h})\end{array}$ \\
\hline V0 & $73,37-0 \%$ & $9,28-0 \%$ & $0,294-0 \%$ \\
V1 & $78,24-7 \%$ & $9,50-2 \%$ & $0,297-1 \%$ \\
V2 & $92,34-26 \%$ & $10,73-16 \%$ & $0,310-5 \%$ \\
V3 & $104,7-43 \%$ & $11,07-19 \%$ & $0,326-11 \%$ \\
V4 & $121,0-65 \%$ & $11,87-28 \%$ & $0,344-17 \%$ \\
V5 & $133,7-83 \%$ & $11,93-29 \%$ & $0,356-20 \%$ \\
\hline
\end{tabular}

de precipitação. Também são indicados os vetores de vento, sendo possível identificar escoamentos em sentidos contrários junto à superfície, sob o sistema. Os resultados evidenciam a produção de rajadas horizontais associadas à precipitação, como mecanismo de intensificação dos fluxos superficiais.

A variável MoMe $(|\bar{V}|)$ é o módulo da média da velocidade e MeMo $(\overline{|V|})$ é a média do módulo da velocidade no domínio na primeira camada. Portanto, para esse domínio e essa hora especificamente, a razão entre as variáveis MeMo $(2,46 \mathrm{~m} / \mathrm{s})$ e MoMe $(0,16 \mathrm{~m} / \mathrm{s})$, que corresponde ao fator de intensificação dos fluxos, atinge um valor de 15,37, ou seja, as rajadas promovidas nesse sistema convectivo nessa porção do domínio produziram fluxos de calor 15 vezes maiores do que o estimado, apenas considerando os proporcionais ao módulo do vento de grande escala.

\subsection{Relação entre a intensificação dos fluxos, taxa de precipitação e vento de grande escala}

No desenvolvimento de uma parametrização do efeito de rajadas, o primeiro aspecto a ser considerado é se há correlação entre o fator de intensificação e a taxa de precipitação convectiva no CEM. A Figura 6 mostra a média dessas duas variáveis ao longo de um trecho de 100 horas da simulação V0 no domínio completo $(1024 \mathrm{~km})$. A figura sugere a existência de uma correlação entre o fator de intensificação com a taxa de precipitação, já que as duas variáveis encontram-se predominantemente em fase, mostrando que as rajadas promovidas pelas nuvens precipitantes perturbam o campo de velocidade no domínio do CEM, favorecendo um aumento dos fluxos de calor latente e sensível.

O domínio do CEM pode ser considerado um análogo a uma coluna de um modelo de circulação geral. No entanto, um domínio muito extenso, de $1024 \mathrm{~km}$ representa um espaçamento de grade muito grande, mesmo para os padrões dos modelos globais de baixa resolução usados no presente. Como pretende-se construir uma parametrização que considere o fato de que para resoluções mais finas (poucas dezenas de quilômetros), o efeito das rajadas pode começar a ser marginalmente resolvido, adota-se a estratégia de subdividir o domínio do CEM em dois, para mimetizar um MCGA com resolução de $512 \mathrm{~km}$; em quatro, para um MCGA de resolução de 256 km; e assim por diante.

As Tabelas 2 e 3 mostram as correlações de Spearman e de Pearson entre a precipitação e o fator de intensificação para as várias simulações. À medida que se diminui o número de colunas do CEM (usadas para mimetizar uma única coluna de um MCGA), as correlações entre as séries temporais do fator de intensificação e da taxa de precipitação reduzem consideravelmente. De modo geral, a taxa de precipitação, para espaçamentos de MCGA maiores ou iguais a $64 \mathrm{~km}$, apresenta correlações de Spearman de 0,40 ou mais, com exceção da simulação V0, para a qual essa correlação é modesta para quase todas os casos, como mostra a tabela 4. Esses resultados sugerem que, de fato, é possível representar a intensificação dos fluxos de forma simples, a partir da precipitação, pelo menos para modelos globais de baixa resolução.

As correlações entre o fator de intensificação e o módulo da velocidade média também foram calculados para cada espaçamento simulado de um MCGA por agrupamento de colunas do CEM, unindo todas as simulações. Observa-se claramente que há uma dependência do fator de intensificação com a velocidade média, com correlações de Spearman entre -0,465 (16km) e -0,606 (1024km) e correlações de Pearson entre -0,319 (16 km) e -0,732 $(1024 \mathrm{~km})$. Novamente, isto sugere que o fator de intensificação, ao decrescer na medida em que se aumenta a velocidade do vento, pode ser parametrizado em função desta variável, especialmente para modelos de baixa resolução. Os valores anticorrelacionados entre o fator de intensificação com a velocidade concordam com a hipótese de formulação da parametrização e com trabalhos formulados por Redelsperger et al. (2000).

Assim, conclui-se que se pode tentar construir uma parametrização para a intensificação dos fluxos que seja 


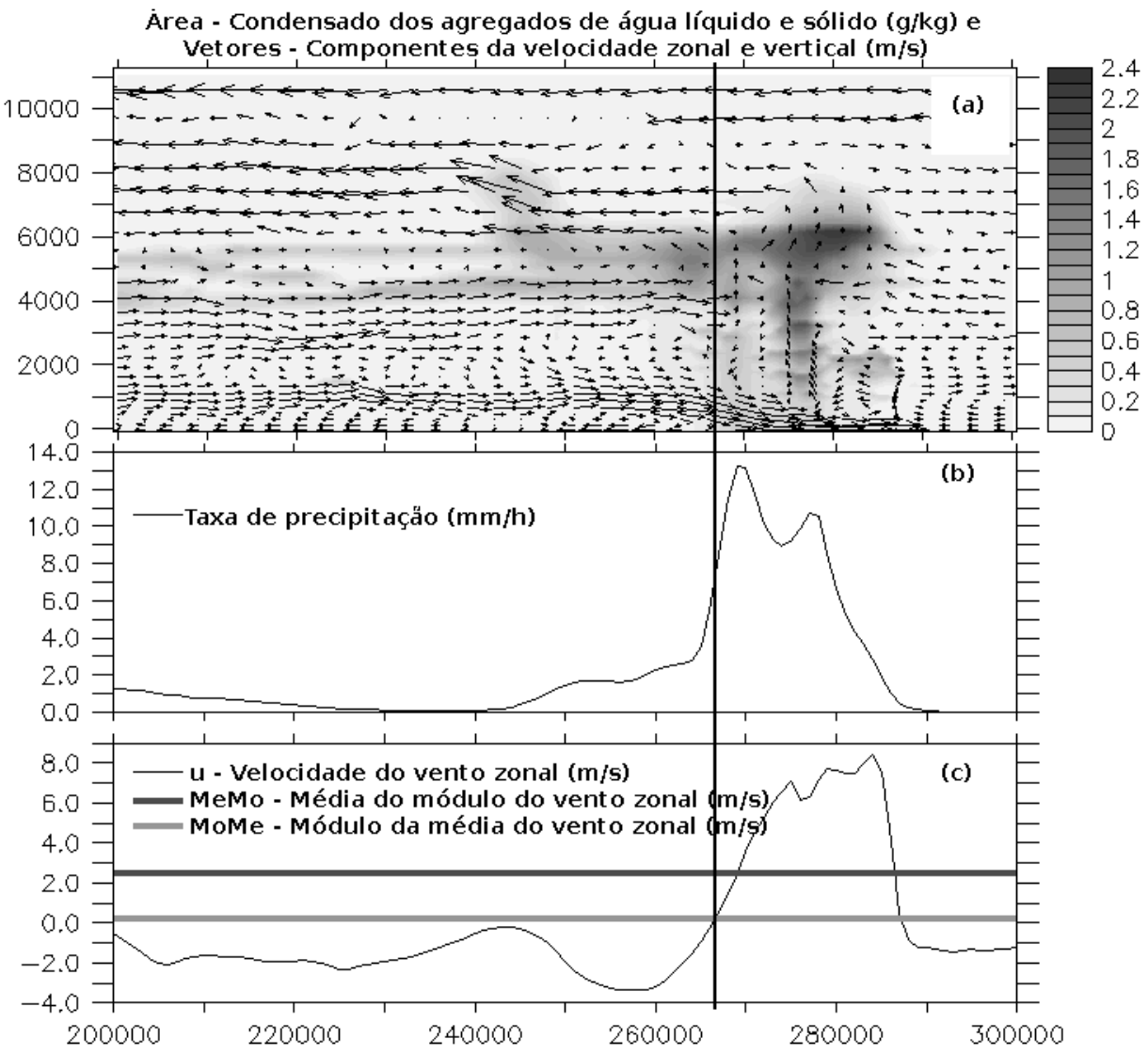

Figura 5 - Dados da simulação com o perfil vertical de vento forçado a 0 m/s (grupo V0), hora 636, no intervalo de 200 a $300 \mathrm{~km}$ mostrando o (a) condensando dos agregados de água em $\mathrm{g} / \mathrm{kg}$, (b) a taxa de precipitação em $\mathrm{mm} / \mathrm{h}$ e (c) a velocidade do vento zonal: A linha contínua cinza clara representa o módulo da velocidade média no domínio e a linha contínua cinza escura representa a média do módulo da velocidade no domínio.

Fator de realce de fluxos e taxa de precipitacao $(\mathrm{mm} / \mathrm{h})$

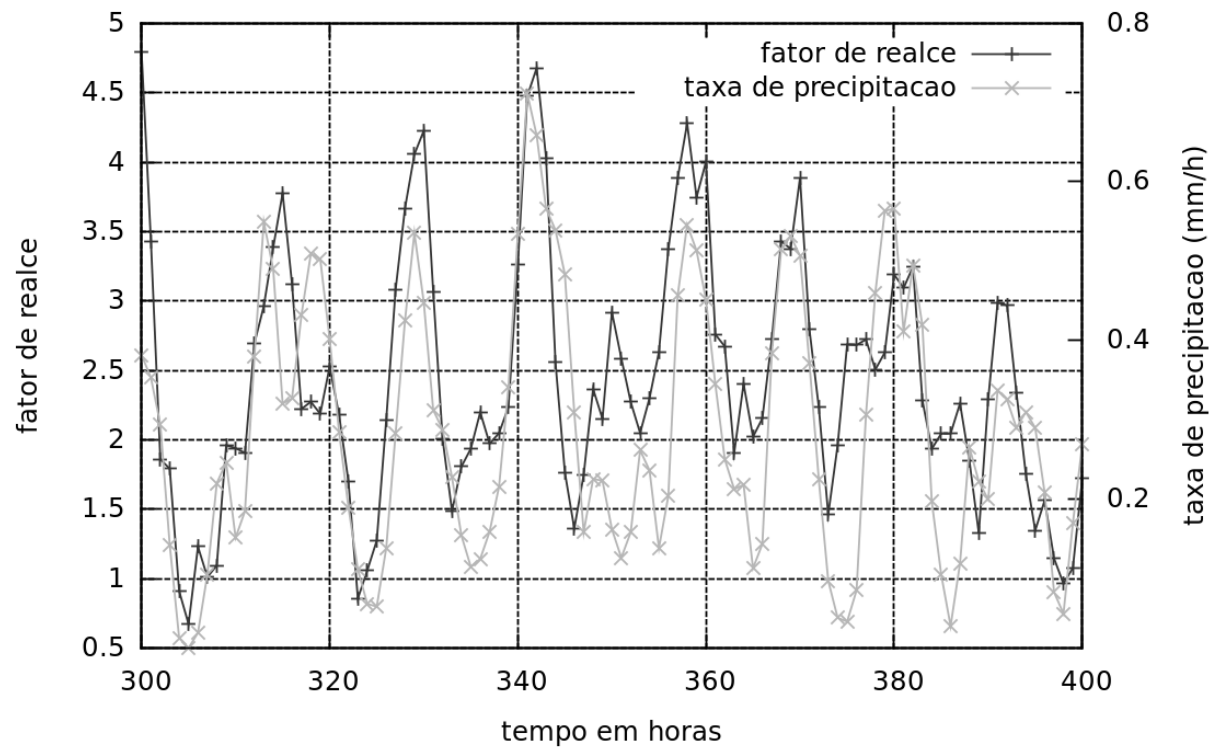

Figura 6 - Fator de intensificação dos fluxos versus taxa de precipitação (mm/h) para a simulação V0 durante parte da simulação. 
Tabela 2 - Índices calculados da correlação de Spearman entre o fator de intensificação e a taxa de precipitação. Valores acima de 0,40 são destacados em negrito.

\begin{tabular}{ccccccc}
\hline $\begin{array}{c}\text { Resolução } \\
(\mathbf{k m})\end{array}$ & V0 & V1 & V2 & V3 & V4 & V5 \\
\hline $\mathbf{1 0 2 4}$ & $\mathbf{0 , 6 6 5}$ & $\mathbf{0 , 6 2 6}$ & $\mathbf{0 , 5 3 1}$ & $\mathbf{0 , 5 2 3}$ & $\mathbf{0 , 4 3 3}$ & $\mathbf{0 , 4 0 2}$ \\
$\mathbf{5 1 2}$ & $-0,001$ & 0,060 & 0,301 & $\mathbf{0 , 5 7 6}$ & $\mathbf{0 , 5 0 1}$ & $\mathbf{0 , 4 2 8}$ \\
$\mathbf{2 5 6}$ & 0,053 & 0,090 & 0,021 & 0,110 & 0,034 & 0,385 \\
$\mathbf{1 2 8}$ & 0,007 & 0,056 & 0,038 & 0,018 & 0,045 & 0,145 \\
$\mathbf{6 4}$ & 0,016 & 0,041 & 0,033 & 0,059 & 0,089 & 0,111 \\
$\mathbf{3 2}$ & 0,044 & 0,058 & 0,024 & 0,048 & 0,051 & 0,084 \\
$\mathbf{1 6}$ & 0,051 & 0,026 & 0,043 & 0,037 & 0,006 & 0,008 \\
\hline
\end{tabular}

função da precipitação, do vento superficial de grande escala e, possivelmente, da resolução do modelo. Uma parametrização para este efeito já foi desenvolvida (Mondon e Redelsperger, 1998; Redelsperger et al., 2000), entretanto, há evidências que a resolução horizontal também contribui no efeito (Zeng et al., 2002).

Esta evidência de dependência do efeito de intensificação dos fluxos superficiais de calor com o espaçamento de grade, já foi obtido através de um fator de ajuste desenvolvido através de resultados usando CEMs com dados dos experimentos GATE I, GATE II e TOGA (Zeng et al., 2002).

Além de comprovação por resultados numéricos experimentais, esta dependência também pode ser comprovada por uma simples relação algébrica. Dada que a razão entre as velocidades é expressa por $\left(\overline{|V|} /|\bar{V}|\right.$ ), que existam $N_{x}$ e $N_{y}$ pontos de grades (domínio de pontos) e um campo de velocidade de vento perturbado por uma componente vertical de velocidade (uma rajada de ventos de uma nuvem convectiva precipitante). Ao determinar o valor médio do valor absoluto de velocidade de vento $(\overline{|V|})$ de cada ponto de grade sobre os $N_{x}$ e $N_{y}$ pontos e dividindo pelo valor absoluto da velocidade média do vento $(\bar{V} \mid)$ de cada ponto de grade sobre os $N_{x}$ e $N_{y}$ pontos. Espera-se, por solução analítica, que para fracos campos de velocidade do vento os valores desta razão exceda 1 , ou seja $\overline{|V|} /|\bar{V}| \geq 1$ e para fortes campos de velocidade de vento estes valores sejam aproximadamente igual a 1 , ou seja $\overline{|V|} /|\bar{V}| \cong 1$, isto é, dado que o campo de velocidade não seja homogêneo.

\subsection{Fitting dos dados e obtenção da equação da amplificação dos fluxos}

Dentre as várias possíveis maneiras de ajustar os dados do CEM, é preciso considerar uma que seja fisicamente plausível para situações limite, isto é, a intensificação não deve ocorrer na ausência de precipitação, nem para valores muito grandes do vento de grande escala. Daí, uma equação que se adapta a estes limites é.

$$
\varepsilon(|\bar{V}|, P)=a(\Delta x) P^{b(\Delta x)}|\bar{V}|^{d(\Delta x)}
$$

onde $P$ é a taxa de precipitação convectiva, $|\bar{V}|$ é o módulo da velocidade média no domínio, $\Delta \mathrm{x}$ e a $(\Delta \mathrm{x}), \mathrm{b}(\Delta \mathrm{x})$ e $\mathrm{d}(\Delta \mathrm{x})$ são parâmetros calculados devido à resolução espacial. Dentre estes coeficientes, $\mathrm{b}(\Delta \mathrm{x})$ deve ser positivo e $\mathrm{d}(\Delta \mathrm{x})$ negativo a fim de obedecer às condições limite. Um exemplo da obtenção desse

Tabela 3 - Como na Tabela 2, mas para correlação de Pearson.

\begin{tabular}{ccccccc}
\hline $\begin{array}{c}\text { Resolução } \\
(\mathbf{k m})\end{array}$ & V0 & V1 & V2 & V3 & V4 & V5 \\
\hline $\mathbf{1 0 2 4}$ & $\mathbf{0 , 6 6 5}$ & $\mathbf{0 , 6 2 6}$ & $\mathbf{0 , 5 3 1}$ & $\mathbf{0 , 5 2 3}$ & $\mathbf{0 , 4 3 3}$ & $\mathbf{0 , 4 0 2}$ \\
$\mathbf{5 1 2}$ & $-0,001$ & 0,060 & 0,301 & $\mathbf{0 , 5 7 6}$ & $\mathbf{0 , 5 0 1}$ & $\mathbf{0 , 4 2 8}$ \\
$\mathbf{2 5 6}$ & 0,053 & 0,090 & 0,021 & 0,110 & 0,034 & 0,385 \\
$\mathbf{1 2 8}$ & 0,007 & 0,056 & 0,038 & 0,018 & 0,045 & 0,145 \\
$\mathbf{6 4}$ & 0,016 & 0,041 & 0,033 & 0,059 & 0,089 & 0,111 \\
$\mathbf{3 2}$ & 0,044 & 0,058 & 0,024 & 0,048 & 0,051 & 0,084 \\
$\mathbf{1 6}$ & 0,051 & 0,026 & 0,043 & 0,037 & 0,006 & 0,008 \\
\hline
\end{tabular}


Tabela 4 - Índices de correlação de Spearman e Pearson calculado para o fator de intensificação e o módulo da velocidade média com todos os grupos de velocidade em cada domínio do CEM.

\begin{tabular}{ccc}
\hline \multirow{2}{*}{$\begin{array}{c}\text { Resolução } \\
(\mathbf{k m})\end{array}$} & \multicolumn{2}{c}{ Tipo de Correlação } \\
\cline { 2 - 3 } & Spearman & Pearson \\
\hline $\mathbf{1 0 2 4}$ & $\mathbf{- 0 , 6 0 6}$ & $\mathbf{- 0 , 7 3 2}$ \\
$\mathbf{5 1 2}$ & $\mathbf{- 0 , 8 5 5}$ & $\mathbf{- 0 , 6 3 1}$ \\
$\mathbf{2 5 6}$ & $\mathbf{- 0 , 7 8 1}$ & $\mathbf{- 0 , 5 7 7}$ \\
$\mathbf{1 2 8}$ & $\mathbf{- 0 , 7 0 6}$ & $\mathbf{- 0 , 5 1 4}$ \\
$\mathbf{6 4}$ & $\mathbf{- 0 , 6 2 6}$ & $\mathbf{- 0 , 4 4 9}$ \\
$\mathbf{3 2}$ & $\mathbf{- 0 , 5 4 4}$ & $-0,381$ \\
$\mathbf{1 6}$ & $\mathbf{- 0 , 4 6 5}$ & $-0,319$ \\
\hline
\end{tabular}

ajuste para o caso da resolução de $1024 \mathrm{~km}$, incluindo todos os dados das simulações de V0 a V5 é mostrado na Figura 7. Os valores obtidos dos parâmetros para cada resolução são apresentados na Tabela 5, juntamente com a correlação entre a curva de ajuste e os dados do CEM. Percebe-se claramente que para cada fitting existem valores elevados para o coeficiente de determinação $\left(\mathrm{r}^{2}\right)$, estando na faixa de $0,47(32 \mathrm{~km})$ até atingir seu valor máximo de $0,88(1024 \mathrm{~km})$, o que garante uma representação adequada da intensificação dos fluxos desde modelos globais de resolução muito baixa até modelos de mesoescala.

A última etapa na obtenção da equação do esquema de intensificação de fluxos é chegar em fórmulas para os parâmetros $a, b$ e $d$, para uma resolução qualquer. Estas equações foram obtidas, com coeficientes de determinação elevado para todos os parâmetros. A equação, ao considerar também a resolução do modelo para o fator de intensificação é apresentada a seguir:

$$
\begin{aligned}
\varepsilon= & 0,232 \ln (1+0,8771 \Delta x) P^{\left(2,17 E-16 \Delta x^{5,034}+0,2346\right)} \\
& |\bar{V}|^{\left(-8,66 E-2 \Delta x^{0,3713}-0,4976\right)}
\end{aligned}
$$

sendo $\Delta \mathrm{x}$ a resolução do modelo bidimensional, $\mathrm{P}$ a taxa de precipitação em milímetros por hora e $\overline{|V|}$ a velocidade do vento resolvida na primeira camada do modelo $(39 \mathrm{~m})$.

Os parâmetros ajustados nesta parametrização não são comparáveis com as outras formulações de Redelsperger et al. (2000), Wu e Guimond (2006) e Zeng et al. (2002), pois, o efeito da intensificação, neste caso, é tratado através de um fator $(\varepsilon)$ e nos outros a velocidade de vento $(\overline{|V|})$ é parametrizado. Mas, em geral, as dependências entre as variáveis mantiveram comportamento semelhante entre o efeito da intensificação.

\section{CONCLUSÕES}

Um CEM foi forçado com cinco perfis verticais de velocidade do vento, transladados a partir do perfil vertical médio de dados do TOGA-COARE sobre o Oceano Pacífico ocidental, a fim de se investigar a intensificação dos fluxos de calor devido às rajadas associadas a correntes descendentes produzidas por sistemas convectivos. O objetivo seguinte é construir uma parametrização física desse efeito, em função da taxa de precipitação, do módulo da velocidade média e da própria resolução de um modelo bidimensional para ser usada em MCGA e modelos de mesoescala.

O modelo representou satisfatoriamente as variáveis: CAPE, água precipitável, fluxos de calor latente e sensível através

Fator de intensificação

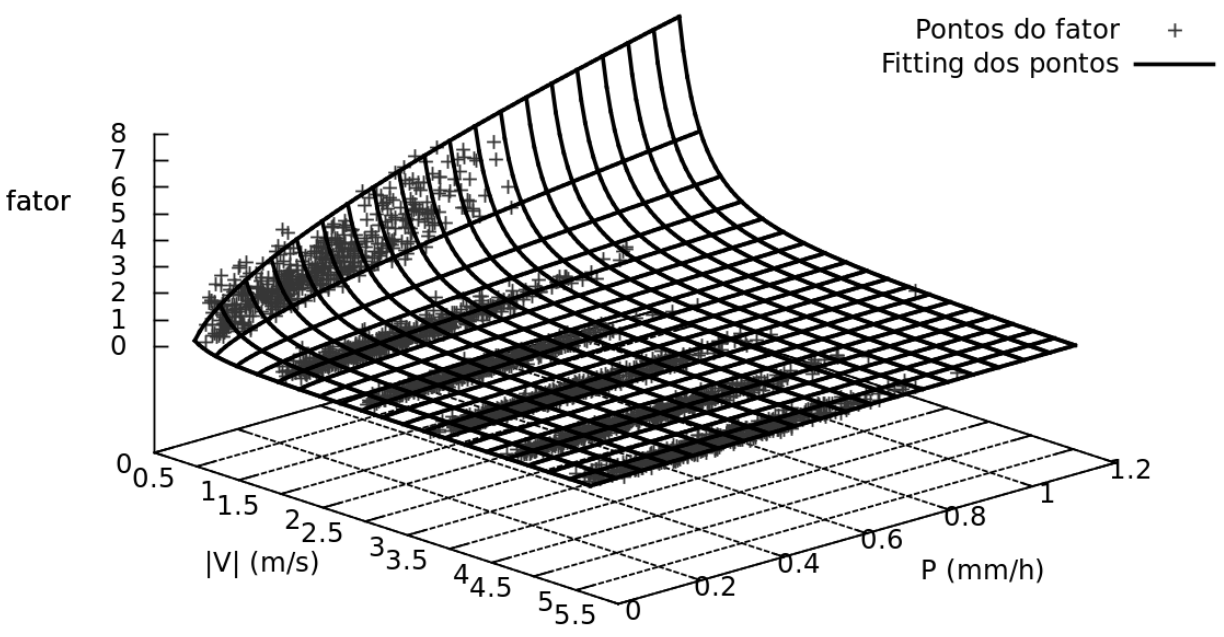

Figura 6 - Fator de intensificação dos fluxos versus taxa de precipitação (mm/h) para a simulação V0 durante parte da simulação. 
Tabela 5 -Parâmetros obtidos da regressão realizada em todos os grupos de velocidade das variáveis: velocidade média na primeira camada do modelo e da taxa de precipitação por cada resolução.

\begin{tabular}{ccccc}
\hline Resolução (km) & $\boldsymbol{a}(\Delta \boldsymbol{x})$ & $\boldsymbol{b}(\Delta \boldsymbol{x})$ & $\boldsymbol{d}(\Delta \boldsymbol{x})$ & $\mathbf{R}^{\mathbf{2}}$ \\
\hline $\mathbf{1 0 2 4}$ & 1,697 & 0,543 & $-1,644$ & $\mathbf{0 , 8 8 2}$ \\
$\mathbf{5 1 2}$ & 1,281 & 0,241 & $-1,348$ & $\mathbf{0 , 8 0 5}$ \\
$\mathbf{2 5 6}$ & 1,168 & 0,211 & $-1,197$ & $\mathbf{0 , 7 5 5}$ \\
$\mathbf{1 2 8}$ & 1,154 & 0,240 & $-1,022$ & $\mathbf{0 , 6 7 0}$ \\
$\mathbf{6 4}$ & 1,008 & 0,238 & $-0,897$ & $\mathbf{0 , 5 6 2}$ \\
$\mathbf{3 2}$ & 0,798 & 0,248 & $-0,825$ & $\mathbf{0 , 4 7 0}$ \\
$\mathbf{1 6}$ & 0,591 & 0,239 & $-0,730$ & 0,369 \\
\hline
\end{tabular}

dos estados de quase-equilíbrio. Dado que a configuração do modelo foi de fronteiras periódicas implicando na conservação de massa, que em outras palavras, contribuiu para que o modelo fosse capaz de atingir estes estados. Esses estados concordam com experiências anteriores quando se utilizam o RAMS como um CEM (Costa, 2004a, 2004b; Olsson et al., 1998).

Um resultado encontrado, onde não há citações comparáveis, que quando um CEM é forçado com diferente velocidade de vento de grande escala, parece existir um tempo para que o CEM atinja os estados de quase equilíbrio. Particularmente, em situações cujo vento de grande escala o modelo requer um maior tempo para o fim do período transiente.

Isto sugere que em situações com fracos campos de velocidade de vento de grande escala próximo à superfície, a intensificação dos fluxos atua como um mecanismo dinâmico que contribui para aumentar a quantidade energética na atmosfera. Neste sentido, quanto ao aspecto de modelagem de processos atmosféricos, os MCGA que não resolvem nuvens convectivas precipitantes na escala da subgrade deixam de remover mais fluxos turbulentos de calor para a atmosfera, por não resolver a intensificação da velocidade do vento devido às rajadas associadas às nuvens convectivas precipitantes próximas à superfície.

Através dos coeficientes de correlação calculados entre o fator de intensificação e a velocidade média do vento de grande escala foi possível verificar que o efeito da intensificação dos fluxos superficiais de calor é inversamente proporcional ao vento de grande escala, e neste sentido, em situações de velocidade de vento elevados valores de fluxos superficiais de calor são removido da superfície. Nesta situação, o principal mecanismo que contribui na intensificação dos fluxos superficiais de calor não é a velocidade média do vento, mas a média do módulo das velocidades, pois independente do sentido do fluxo do fluído atmosférico, os fluxos turbulentos de calor são calculados pelo módulo da velocidade do vento.

Foi notado que existe uma defasagem entre os picos do fator de intensificação dos fluxos superficiais de calor e a taxa de precipitação, embora sejam bem correlacionados. Neste sentido, há um retardo no máximo do fator de intensificação dada a ocorrência da precipitação de uma nuvem convectiva. Isto é coerente, dado que após a precipitação, parte do material precipitante evapora e então uma quantidade de calor latente de evaporação é removida da atmosfera. Essa remoção de calor contribui para a diminuição local da temperatura e por fim promovendo a descendência local dessas massas de ar através das rajadas de ventos de nuvens convectivas precipitantes.

No fitting dos dados, foi verificado que, para resoluções acima de $32 \mathrm{~km}$, correlações razoáveis são encontradas, a sugerir que modelos na faixa de resolução de $512 \mathrm{~km}$ a $32 \mathrm{~km}$ podem ter a intensificação de fluxos por rajadas bem representada por uma parametrização simples.

Atualmente, estão sendo realizadas simulações 3D para verificar se a intensificação dos fluxos de calor possui o mesmo comportamento verificado neste trabalho, com o objetivo de obter uma versão aprimorada do esquema aqui apresentado. Ainda como trabalho futuro se pretende aplicar esse esquema 2D (e sua versão baseada em simulações 3D) num modelo de coluna individual, Single-Colunm Model (SCM), realizar os testes desta parametrização e, após a validação desses testes, as implementar em um MCGA.

\section{AGRADECIMENTOS}

O primeiro autor agradece a bolsa de demanda social concedido pela Coordenação de Aperfeiçoamento de Pessoal de Nível Superior (CAPES). Os autores agradecem ao Conselho Nacional de Desenvolvimento Científico e Tecnológico (CNPQ) pelo suporte financeiro concedido para a atual estrutura de computadores que possui o programa de Mestrado em Ciências Físicas Aplicadas (MACFA). Em especial, os autores agradecem aos revisores anônimos que em muito contribuíram, através de uma discussão intensa, para a finalização deste trabalho.

\section{REFERÊNCIAS BIBLIOGRÁFICAS}

ARAKAWA, A.; SCHUBERT, W. H. Interactions of a Cumulus Cloud Ensemble with Large-Scaler Environment, Part I. Journal of the Atmospheric Sciences, v. 31, p. 674-702, 1974.

BRADLEY, E. F.; COPPIN, P. A.; GODFREY, J. S. Measurements of sensible and latent heat flux in the western equatorial Pacific Ocean. Journal of Geophysical Research: Oceans, v. 96, n. S01, p. 3375-3389, 1991.

CHEN, C.; COTTON, W. R. The Physics of the Marine Stratocumulus-Capped Mixed Layer. Journal of the Atmospheric Sciences, v. 44, n. 20, p. 2951-2977, 1987. 
COSTA, A. A. Cloud-Resolving-Model of Air-Sea interactions over the Western Pacific. Fort Collins, Colorado: (Departament of Atmospheric Science) Colorado State University, 2000.

COSTA, A. A. Cloud-ensemble simulations of atmospheric convection over the ocean part i: model description, coupled and uncoupled simulations of a TOGA-COARE Case. Revista Brasileira de Meteorologia, v. 19, n. 3, p. 227-236, 2004a.

COSTA, A. A. Cloud-ensemble simulations of atmospheric convection over ocean part ii: numerical experiments with prescribed, inhomogeneous sea surface temperatures. Revista Brasileira de Meteorologia, v. 19, n. 3, p. 243-250, 2004b.

COSTA, A. A.; COTTON, W. R.; WALKO, R. L.; A, R.; SR, P. Coupled Ocean-Cloud-Resolving Simulations of the Air-Sea Interaction over the Equatorial Western Pacific. Journal of the Atmospheric Sciences, v. 58, p. 3357-3376, 2001.

GRABOWSKI, W. W; WU, X.; MONCRIEFF, M. W. Cloud Resolving Modeling of Tropical Cloud Systems during Phase III of GATE. Part III: Effects of Cloud Microphysics. Journal of the Atmospheric Sciences, v. 56, p. 2384-2402, 1999.

GRABOWSKI, WOJCIECH W.; WU, X.; MONCRIEFF, M. W. Cloud-Resolving Modeling of Tropical Cloud Systems during Phase III of GATE. Part I: Two-Dimensional Experiments. Journal of the Atmospheric Sciences, v. 53, n. 24, p. 3684-3709, 1996.

GRABOWSKI, WOJCIECH W.; WU, X.; MONCRIEFF, M. W.; HALL, W. D. Cloud-Resolving Modeling of Cloud Systems during Phase III of GATE. Part II: Effects of Resolution and the Third Spatial Dimension. Journal of the Atmospheric Sciences, v. 55, n. 21, p. 3264-3282, 1998.

MITSUTA, Y.; TSUKAMOTO, O. Drag Coefficients in Light Wind. Bulletin of the Disaster Prevention Research Institute Kyoto University, v. 28, p. 25-32, 1978.

MONCRIEFF, M. W.; STEVEN, K. K.; GREGORY, D.; REDELSPERGER, J.-L.; TAO, WEI-KUO. Gewex cloud system study (gcss) working group 4: precipitating convective cloud systems. Bulletin of the American Meteorological Society, v. 78, p. 831-845, 1997.

MONDON, S.; REDELSPERGER, J.-L. A study of a fair weather boundary layer in TOGA-COARE: Parametrization of surface fluxes in large-scale and regional models for light wind conditions. Boundary-Layer Meteorology, v. 88, p. 47-76, 1998.

NORONHA, A. W. T.; COSTA, A. A.; SANTOS, A. C. S.; SOUSA, A. C. T. DE; SANTOS, C. C. DOS. Análise dos fluxos de calor de superfície variando o diâmetro de gota da chuva usando um modelo de nuvem resolvida. Mudanças Climáticas e seus impactos em Áreas Urbanas. Anais... João Pessoa - PB: [s.n.]. Disponível em: <http:// sic2011.com/sic/arq/74193820566557419382056.pdf >, 2011

OLSSON, P. Q.; HARRINGTON, JERRY Y; FEINGOLD, G.; COTTON, W. R.; KREIDENWEIS, S. M. Exploratory cloud-resolving simulations of boundary-layer Arctic stratus clouds: Part I: Warm-season clouds. Atmospheric Research, v. 47-48, n. 0, p. 573-597, 1998.

PEIXOTO, J. P.; OORT, A. H. Radiation Balance. Physics of climate. New York: American Institute of Physics, p. 91-94, 1991.

PINHEIRO, A. DE S.; COSTA, A. A. Sensibilidade de Conjuntos de Nuvens a Variações de Parâmetros Microfísicos. Parte I - Simulação de Controle. Revista Brasileira De Meteorologia, v. 28, n. 1, p. 13-24, 2013.

RANDALL, D. A.; XU, K.-M.; SOMERVILLE, R. J. C.; IACOBELLIS, S. Single-Column Models and Cloud Ensemble Models as Links beteween Observations and Climate Models. Journal of Climate, v. 9, p. 1683-1696, 1996.

REDELSPERGER, J.-L.; GUICHARD, F.; MONDON, S. A Parameterization of Mesoscale Enhancement of Surface Fluxes for Large-Scale Models. Journal of Climate, v. 13, p. 402-421, 2000.

SMAGORINSKY, J. General Circulation Experiments with the primitive equations. Monthly Weather Review, v. 91, n. 3, p. 99-164, 1963.

TREMBACK, K. E.; JONES, P. D.; AMBENJE, P.; et al. Observations: Surface and Atmospheric Climate Change. Climate Change 2007: The Physical Science Basis. Contribution of Working Group I to the Fourth Assessment Report of the Intergovernmental Panel on Climate Change. New York: Cambridge University Press, 2007. p. 995.

WALKO, R. L.; COTTON, W. R.; MEYERS, M. P.; HARRINGTON, J. Y. New RAMS clou microphysics parameterizations Part I:the single-moment scheme. Atmospheric Research, v. 38, p. 29-62, 1995.

WU, X.; GUIMOND, S. Two-and three-dimensional cloudresolving model simulations of the mesoscale enhancement of surface heat fluxes by precipitating deep convection. Journal of Climate, v. 19, p. 139-149, 2006.

WU, X.; LI, X. A review of cloud-resolving model studies of convective processes. Advances in Atmospheric Sciences, v. 25, n. 2, p. 202-212, 2008.

ZENG, X.; ZHANG, Q.; JOHNSON, D.; TAO, W-K. Parameterization of Wind Gustiness for the Computation of Ocean Surface Fluxes at Different Spatial Scales. Monthly Weather Review, v. 130, n. 8, p. 2125-2133, 2002. 